\title{
Epidemiological and clinical characteristics of severe fever with thrombocytopenia syndrome (SFTS) in China: an integrated data analysis
}

\author{
C.-T. GUO ${ }^{1,2} \dagger$, Q.-B. LU ${ }^{3} \dagger$, S.-J. DING ${ }^{4} \dagger$, C.-Y. HU ${ }^{1}$, J.-G. HU ${ }^{1}$, Y. WO ${ }^{1}$,

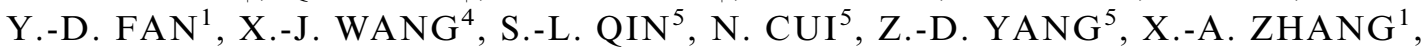 \\ W. LIU $^{1,2 *}$ AND W.-C. CAO ${ }^{1}$ \\ ${ }^{1}$ State Key Laboratory of Pathogen and Biosecurity, Beijing Institute of Microbiology and Epidemiology, Beijing, \\ P.R. China \\ ${ }^{2}$ Graduate School of Anhui Medical University, Hefei, P.R. China \\ ${ }^{3}$ School of Public Health, Peking University, Beijing, P.R. China \\ ${ }^{4}$ Department for Virus Infectious Diseases Control and Prevention, Shandong Center for Disease Control and \\ Prevention, Shandong Provincial Key Laboratory of Communicable, Disease Control and Prevention, Jinan, \\ Shandong, P.R. China \\ ${ }^{5}$ The 154 Hospital, People's Liberation Army, Xinyang, P.R. China
}

Received 5 August 2015; Final revision 1 October 2015; Accepted 7 October 2015; first published online 6 November 2015

\section{SUMMARY}

Severe fever with thrombocytopenia syndrome (SFTS) is an emerging infectious disease that was caused by a novel bunyavirus, SFTSV. The study aimed to disclose the epidemiological and clinical characteristics of SFTSV infection in China so far. An integrated clinical database comprising 1920 SFTS patients was constructed by combining first-hand clinical information collected from SFTS sentinel hospitals $(n=1159)$ and extracted data $(n=761)$ from published literature. The considered variables comprised clinical manifestations, routine laboratory tests of acute infection, hospitalization duration and disease outcome. SFTSV-IgG data from 19119 healthy subjects were extracted from the published papers. The key clinical variables, case-fatality rate (CFR) and seroprevalence were estimated by meta-analysis. The most commonly seen clinical manifestations of SFTSV infection were fever, anorexia, myalgia, chill and lymphadenopathy. The major laboratory findings were elevated lactate dehydrogenase, aminotransferase, followed by thrombocytopenia, lymphocytopenia, elevated alanine transaminase and creatine kinase. A CFR of $12 \cdot 2 \%$ was estimated, significantly higher than that obtained from national reporting data, but showing no geographical difference. In our paper, the mortality rate was about 1.9 parts per million. Older age and longer delay to hospitalization were significantly associated with fatal outcome. A pooled seroprevalence of $3 \cdot 0 \%$ was obtained, which increased with age, while comparable for gender. This study represents a clinical characterization on the largest group of SFTS patients up to now. A higher than expected CFR was obtained. A wider spectrum of clinical index was suggested to be used to identify SFTSV infection, while the useful predictor for fatal outcome was found to be restricted.

Key words: Bunyavirus, China, meta-analysis, severe fever with thrombocytopenia syndrome.

\footnotetext{
* Author for correspondence: Wei Liu, M.D., State Key Laboratory of Pathogen and Biosecurity, Beijing Institute of Microbiology and Epidemiology, 20 DongDa Street, Fengtai District; Beijing 100071, P.R. China.

(Email: lwbime@163.com)

$\dagger$ These authors contributed equally to this work.
} 


\section{INTRODUCTION}

Severe fever with thrombocytopenia syndrome (SFTS) is an emerging infectious disease that was first identified in 2009 in rural areas in central China. A novel bunyavirus (SFTSV) was determined as the cause of SFTS through virus isolation from patients' blood and molecular characterization [1]. By the end of 2013, 14 Chinese provinces had reported almost 2000 laboratory-confirmed SFTS cases [2, 3]. Other Asian countries, including Japan and Korea, have also reported SFTSV-infected patients [4, 5]. STFS's fairly broad distribution, as well as the potential of human-to-human transmission has made this newly emerged zoonosis a severe threat to public health [6-9]. This fact calls for an urgent need to understand its key epidemiological and clinical characteristics, in order to take effective public-health measures to control the epidemic and to recognize which patients require early intervention.

During the 5 years since the discovery of SFTS, although clinical data have been accumulated, studies were only performed in a single medical facility, yielding inconsistent results across studies [6, 10-12]. The case-fatality rate (CFR) ranged marginally from $6.3 \%$ to $30 \%$ in various studies, a difference that cannot be logically explained currently [1, 13-15]. The factors that impact on fatal outcome were widely studied yet lacked replication in independent studies, partially owing to limited sample sizes used. Since first recognition of this novel bunyavirus, SFTS has been added to the list of national notifiable diseases and sentinel hospitals have been established in endemic areas to diagnose and report SFTS cases to China CDC through the China Information System for Diseases Control and Prevention (CISDCP) [16]. However, this system only collected general information regarding patients' age, gender, occupation, residential address, date of disease onset and disease outcome on leaving the hospital. Therefore a nationwide collection of clinical data was lacking and death-related risk analysis based on a large dataset is impracticable. Another query on this emerging infectious disease lies in to what extent the subclinical infection was attained in the healthy population as a whole. The understanding of herd immunity on population level can afford a reliable estimation of the risk posed to the residents in the endemic region. These data, however, are only sporadically reported and varied marginally across studies.

Here we made a systematic study by combining first-hand hospital-based surveillance data and published clinical data, to acquire a comprehensive understanding of the clinical characteristics of the disease, especially the case-fatality data. By performing a literature review and meta-analysis on the seroepidemiological data in the healthy population, the subclinical infection level of SFTSV was estimated. By comparison with the case incidence and case-fatality data, the epidemiological feature of the disease was disclosed.

\section{MATERIALS AND METHODS}

\section{Data source and dataset pooling}

We created an integrated database by combining hospital-based surveillance data and literature review data.

Up to end of 2013, a total of 14 provinces reported 1842 SFTS cases, over half were reported from two provinces (Henan 707, 38.4\% and Shandong 523, $28 \cdot 4 \%$ ). Hospital-based surveillance had been performed in the sentinel hospitals of these two provinces to obtain first-hand clinical information of individual SFTS cases. The patients were recruited and diagnosed according to the standard criteria released by China CDC, i.e. epidemiological exposure, clinical manifestations resembling SFTS and laboratory evidence of SFTSV infection [16]. For these eligible patients, a standardized case investigation form was used to collect the clinical manifestations, routine laboratory tests at acute infection, hospitalization duration and disease outcome. Since there are deaths occurring beyond the observation time during the hospitalization, we used phone call follow-up to acquire the final disease outcome for all recruited patients.

For other provinces where hospital-based surveillance data were unavailable, a literature review was made to collect the relevant information that was already published. Briefly, two main electronic databases (PubMed and China Academic Journals Full-text Database) were systematically searched for all studies published from October 2010 until 31 November 2014, using the following search terms: 'SFTSV' or 'FTLSV' or 'Huaiyangshan virus' in all fields; 'bunyavirus' and 'China' in all fields. We also checked the reference lists of retrieved articles to identify more relevant articles. Only studies recording data on the clinical manifestations and laboratory parameters were included. Each study was read through to collect the information using a standard form, mainly consisting of study date, study design and 


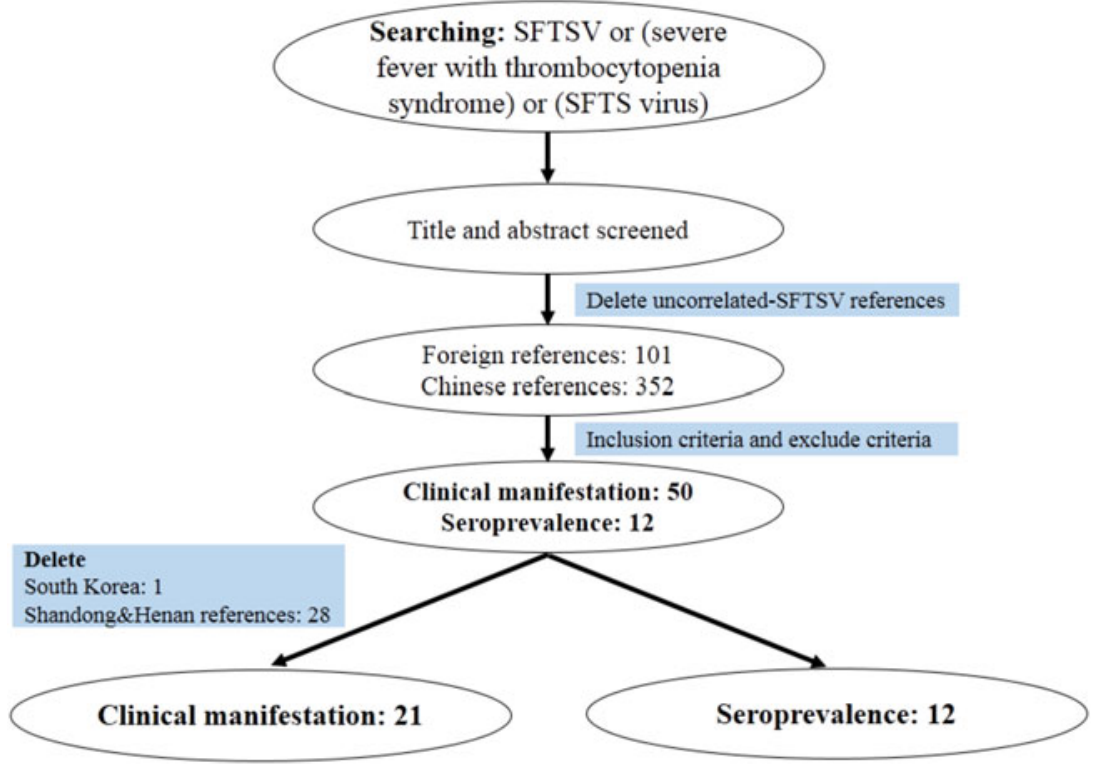

Fig. 1. Flow diagram of the search and review process for creating the integrated dataset.

study area, case diagnosis methods used and demographic information of the studied patients. Clinical manifestations, laboratory parameters expressed by the mean value or the prevalence of abnormal laboratory tests, whichever is available, were recorded. CFR was calculated if the data were presented. Publications were excluded if they reported no human cases of SFTSV infection, reported cases without information indicating the study area, study time or diagnosis method used. Cases that were not diagnosed according to the criteria set by the China CDC or data with cases numbering $<10$ were also excluded.

Two reviewers independently screened and examined the full-text publications by using identical selection criteria and data extraction forms. Any disagreements were resolved by discussion and consensus between the reviewers and other authors. For the articles that contained ambiguous data, the original authors were contacted for confirmation, and if no response was obtained, the ambiguous data were excluded from analyses. The extracted data, together with the clinical data from Henan and Shandong provinces were integrated into one clinical dataset for analysis (Fig. 1). The considered variables were checked and combined by the same reviewers.

Through the literature review, the publications that reported the SFTSV-specific antibody in a healthy population were additionally searched to create a serological dataset. The extracted information included study time, study design, geographical information and seroprevalence. The number of subjects tested and number of subjects positive for SFTSV-specific antibody (by age or gender group when presented) were recorded. Publications were excluded if they reported no information indicating the study location and time, or detailed diagnosed methods used. The same reviewers performed the publication screening and data extraction. These data were combined as a serological dataset (Fig. 1).

For the purpose of comparison, we also acquired national reportable cases infomation during the period from 2010 to 2013 from the CISDCP system [16]. The collected information comprised patients' age, gender, residential address, date of disease onset, date of discharge and outcome on discharge from hospital, which were extracted and combined as an epidemiological dataset. The study was approved by the ethics committees of the Beijing Institute of Microbiology and the attending hospitals.

\section{Statistical analysis}

For categorical variables, counts for cases, and proportions with 95\% confidence intervals (CIs) were presented. For continuous variables, means with standard deviation (S.D.) were calculated. Meta-analysis was conducted using R software (R Foundation, Austria) when $>2$ studies reported the reviewed factor. We tested heterogeneity using the $Q$ statistic and $I^{2}$ test. Pooled values and $95 \%$ CIs were generated from a fixed-effects model 
or from a random-effects model, and each study was weighted by the inverse of that study's variance. We used the fixed-effects model when heterogeneity was not significant and a random-effects model when heterogeneity was evident. Median (range) was converted to mean (s.D.) by using proposed formulae, and interquartile ranges to S.D.s and subgroup values to total values by Cochrane's suggested methods. We assessed publication bias using funnel plots and Egger's regression test (if $>7$ studies were included in the meta-analysis).

\section{RESULTS}

\section{The integrated dataset and included cases}

By using hospital-based surveillance data, a total of 1159 cases that met the standard criteria were recruited (634 in Henan, 525 in Shandong). By applying a literature review, 31 publications that met study criteria for data extraction were used for analysis, including 19 papers reporting clinical data and 12 papers reporting serological data (Fig. 1) $[1,10,12$, 17-44]. All the 19 clinical studies were of crosssectional design, presenting clinical manifestations, laboratory parameters, or both on hospitalized SFTS patients. The sample sizes ranged from 11 to 171, yielding data from 761 laboratory-confirmed SFTS patients. These two datasets were pooled into a single integrated dataset comprising 1920 cases, which is more than 1842 laboratory-confirmed cases that were reported from CISDCP data during the same period. Compared to the national dataset, more cases were obtained in Liaoning province based on the literature review data, which was derived from underreported cases that occurred before 2011 when the CISDCP was not fully operational (Supplementary Table S1). Based on the integrated dataset, the mean age of the patients was $60 \cdot 5 \pm 12.6$ years, and $48.5 \%$ were male. This is highly comparable with the estimate acquired from national reportable cases (age 60.6 $\pm 12 \cdot 1$ years, $48 \cdot 7 \%$ male) (Supplementary Table S1), indicating a good representativeness of the patients in the integrated clinical dataset.

\section{Clinical manifestations and laboratory tests}

The meta-analysis was performed on the integrated clinical dataset. Each variable of clinical manifestation was estimated with a sample size ranging from 669 to 1666 . The main clinical syndrome was fever
Table 1. Main clinical and laboratory parameters at admission for SFTS patients during 2010-2013 in China by meta-analysis

\begin{tabular}{lll}
\hline \hline & $\begin{array}{l}\text { Case number/ } \\
\text { total case } \\
\text { number }\end{array}$ & $\begin{array}{l}\text { Pooled rate } \\
(95 \% \mathrm{CI})\end{array}$ \\
Variables & & \\
\hline $\begin{array}{l}\text { Presence of clinical } \\
\text { manifestations }\end{array}$ & & \\
Fever $>37 \cdot 5{ }^{\circ} \mathrm{C}$ & $1627 / 1666$ & $99 \cdot 7(98 \cdot 8-100)$ \\
Anorexia & $1305 / 1509$ & $86 \cdot 9(80 \cdot 2-92 \cdot 4)$ \\
Myalgia & $903 / 1316$ & $84 \cdot 4(74 \cdot 7-92 \cdot 1)$ \\
Chill & $376 / 784$ & $49 \cdot 1(40 \cdot 9-57 \cdot 3)$ \\
Lymphadenopathy & $391 / 920$ & $40 \cdot 4(32 \cdot 9-48 \cdot 1)$ \\
Diarrhoea & $499 / 1617$ & $38 \cdot 5(31 \cdot 3-45 \cdot 9)$ \\
Vomiting & $541 / 1606$ & $38 \cdot 2(32 \cdot 1-44 \cdot 6)$ \\
Cough & $242 / 815$ & $29 \cdot 7(26 \cdot 6-32 \cdot 8)$ \\
Headache & $403 / 1574$ & $27 \cdot 2(20 \cdot 3-34 \cdot 6)$ \\
Abdominal pain & $208 / 941$ & $25 \cdot 0(17 \cdot 7-33 \cdot 1)$ \\
Expectoration & $163 / 669$ & $24 \cdot 4(21 \cdot 2-27 \cdot 7)$ \\
Confusion & $110 / 750$ & $21 \cdot 1(12 \cdot 1-31 \cdot 9)$ \\
Abnormal laboratory & & \\
tests & & \\
Lactate & & $98 \cdot 8(84 \cdot 2-100)$ \\
dehydrogenase & $551 / 685$ & $98 \cdot 8(57 \cdot 5-100)$ \\
Aminotransferase & $560 / 674$ & $97 \cdot 4(93 \cdot 2-99 \cdot 7)$ \\
Platelet & $1272 / 1523$ & $98 \cdot 5-97 \cdot 8)$ \\
White blood cell & $1206 / 1523$ & $94 \cdot 0-99 \cdot 9)$ \\
Alanine & $427 / 705$ & \\
transaminase & & \\
Creatine kinase & $450 / 673$ & \\
\hline \hline
\end{tabular}

CI, Confidence interval.

(pooled rate $99 \cdot 7 \%, 95 \%$ CI $98 \cdot 8-100$ ), followed by anorexia (pooled rate $86.9 \%, 95 \%$ CI $80 \cdot 2-92 \cdot 4$ ), myalgia (pooled rate $84 \cdot 4 \%$, 95\% CI $74 \cdot 4-92 \cdot 1$ ), chill (pooled rate $49 \cdot 1 \%, 95 \%$ CI $40 \cdot 9-57 \cdot 3$ ) and lymphadenopathy (pooled rate $40 \cdot 4 \%, 95 \%$ CI $32 \cdot 9-48 \cdot 1$ ) (Table 1). The forest plots and funnel plots are summarized in Supplementary Figures S1 and S2.

Each variable of the laboratory tests was estimated with sample size ranging from 673 to 1523 . Six laboratory tests in bunyavirus-infected patients were most commonly seen, i.e. lactate dehydrogenase (LDH) (pooled rate $98 \cdot 8 \%, 95 \%$ CI $84 \cdot 2-100$ ), aminotransferase (AST) (pooled rate $98.4 \%, 95 \%$ CI $82 \cdot 4-100$ ), platelet (PLT) (pooled rate 97.4\%, 95\% CI 93.299.7), white blood cell (WBC) (pooled rate 94.0\%, 95\% CI 88.5-97.8), alanine transaminase (ALT) (pooled rate $89 \cdot 3 \%, 95 \%$ CI $64 \cdot 0-99 \cdot 9$ ), and creatine kinase (CK) (pooled rate $88 \cdot 8 \%, 95 \%$ CI $57 \cdot 5-100$ ) (Table 1). The top five clinical manifestations and abnormal laboratory findings were almost consistently 


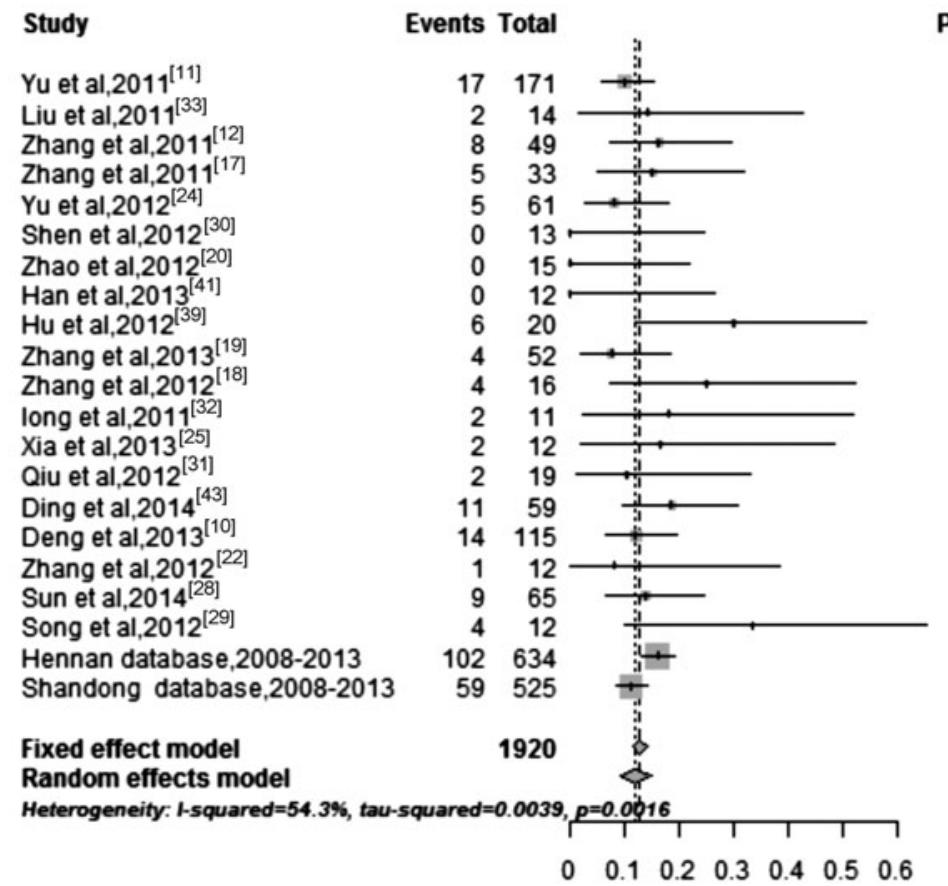

Proportion 95\%-Cl W(fixed) W(random)

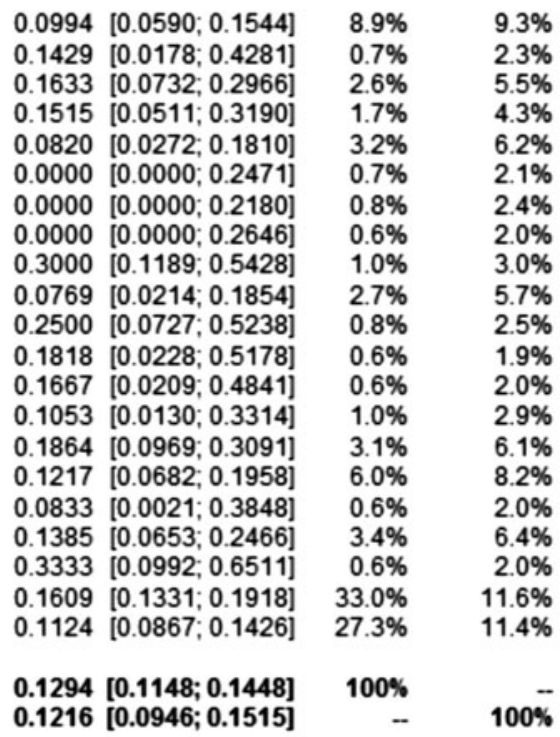

Fig. 2. The forest plot on the case-fatality rate of SFTS patients by the random-effects model.

identified when the data were separately considered regarding the recruiting year and residential region (Supplementary Table S2). The forest plots and funnel plots are summarized in Supplementary Figures S3 and S4.

\section{Estimation of CFR and identification of risk predictors}

Altogether 19 papers provided usable information on fatal cases, from which the CFR ranged from $0 \%$ to $33 \cdot 3 \%$. When these data were integrated for analysis, the mortality rate was estimated to be $12 \cdot 2 \%$ (257/ 1920, 95\% CI 9.5-15·2) (Fig. 2), which was significantly higher than that obtained from the reportable dataset $(8 \cdot 0 \%, 147 / 1842, P<0 \cdot 001)$ (Supplementary Table S3). The funnel plots are summarized in Supplementary Figure S5.

When the CFR was compared with regard to geographical regions, a significantly lower rate in Henan province was found based on the national dataset $(2 \cdot 7 \%)$ than that of the integrated dataset $(16 \cdot 1 \%)$ (Supplementary Table S3). A further investigation on the disease outcome of individual cases revealed that many patients chose to leave hospital against medical advice, and died after being discharged from hospital, which resulted in the under-reporting of death to the CISDCP. The inter-province difference disappeared when the CFR of the integrated dataset was used (Supplementary Table S3). When the case fatality was considered regarding age and gender, higher mortality rates were consistently seen in patients of older age when both meta-analysis and the national dataset were used. Age-dependent increase and annual decrease of CFR were observed for both datasets (linear trend test, both $P<0 \cdot 001$ ), but appeared more frequently in the integrated dataset. Male gender exhibited a higher CFR than female, although this difference did not attain significance (Supplementary Table S3).

The integrated dataset on clinical manifestations was analysed by univariate and multivariate logistic regression models. The results showed that fatal outcome was significantly associated with older age [odds ratio (OR) $1 \cdot 05,95 \%$ CI 1.03-1·06], and longer delay from onset to hospital admission (OR 1·09, 95\% CI 1.02-1·17) (Table 2). The lower PLT counts were demonstrated to be significantly associated with higher case fatality, which disappeared after adjustment for demographic variables by applying multivariate analysis.

\section{Seroprevalence estimation and comparison with case incidence and case fatality}

Altogether 12 studies reporting seroprevalence in apparently healthy populations were included for 
Table 2. The clinical manifestation and laboratory parameters in correlation with fatal SFTSV cases during 2010 2013 using the integrated dataset in China

\begin{tabular}{|c|c|c|c|c|c|c|c|c|}
\hline \multirow[b]{2}{*}{ Variable } & \multirow[b]{2}{*}{ Fatal patients } & \multirow[b]{2}{*}{ Non-fatal patients } & \multicolumn{3}{|l|}{ Crude } & \multicolumn{3}{|c|}{ Adjusted* } \\
\hline & & & OR & $95 \% \mathrm{CI}$ & $P$ & OR & $95 \% \mathrm{CI}$ & $P$ \\
\hline Age, years, mean \pm s.D. & $66 \cdot 1 \pm 10 \cdot 7$ & $59 \cdot 7 \pm 13 \cdot 2$ & $1 \cdot 046$ & $1 \cdot 0301-063$ & $<0 \cdot 001$ & 1045 & $1 \cdot 0271-064$ & $<0 \cdot 001$ \\
\hline Sex, male & $79(51 \cdot 6)$ & $512(48 \cdot 5)$ & $1 \cdot 132$ & $0 \cdot 806-1 \cdot 590$ & $0 \cdot 923$ & 0.954 & $0 \cdot 654-1 \cdot 393$ & $0 \cdot 809$ \\
\hline $\begin{array}{l}\text { Days from onset to } \\
\text { admission, mean } \pm \text { s.D. }\end{array}$ & $6 \cdot 0 \pm 3 \cdot 2$ & $5 \cdot 2 \pm 2 \cdot 9$ & $1 \cdot 072$ & $1 \cdot 021-1 \cdot 125$ & $0 \cdot 005$ & $1 \cdot 092$ & $1 \cdot 022-1 \cdot 167$ & $0 \cdot 010$ \\
\hline Platelet, $10^{9} / 1$ & $64 \cdot 6 \pm 58 \cdot 0$ & $72 \cdot 2 \pm 35 \cdot 7$ & 0.994 & $0 \cdot 989-1 \cdot 000$ & $0 \cdot 034$ & 0.997 & $0 \cdot 992-1 \cdot 003$ & $0 \cdot 321$ \\
\hline White blood cell, $10^{9} / 1$ & $2 \cdot 9 \pm 2 \cdot 4$ & $3 \cdot 0 \pm 2 \cdot 4$ & $0 \cdot 985$ & $0 \cdot 911-1 \cdot 066$ & $0 \cdot 715$ & 0.979 & $0 \cdot 900-1 \cdot 066$ & 0.633 \\
\hline Weakness & $134(87 \cdot 6)$ & $933(88 \cdot 4)$ & $0 \cdot 922$ & $0 \cdot 550-1 \cdot 545$ & 0.759 & $0 \cdot 801$ & $0 \cdot 446-1 \cdot 437$ & $0 \cdot 456$ \\
\hline Lymphadenopathy & $61(40 \cdot 0)$ & $364(34 \cdot 5)$ & $1 \cdot 259$ & $0 \cdot 889-1 \cdot 782$ & $0 \cdot 195$ & $1 \cdot 342$ & $0 \cdot 917-1 \cdot 964$ & $0 \cdot 131$ \\
\hline Conjunctival congestion & $8(5 \cdot 2)$ & $38(3 \cdot 6)$ & $1 \cdot 477$ & $0 \cdot 676-3 \cdot 228$ & $0 \cdot 329$ & $1 \cdot 570$ & $0 \cdot 550-4 \cdot 485$ & $0 \cdot 399$ \\
\hline Gingival bleeding & $87(5 \cdot 2)$ & $31(2 \cdot 9)$ & $1 \cdot 822$ & $0 \cdot 822-4 \cdot 042$ & $0 \cdot 410$ & $1 \cdot 582$ & $0 \cdot 595-4 \cdot 208$ & $0 \cdot 358$ \\
\hline Anorexia & $110(71 \cdot 9)$ & $774(73 \cdot 4)$ & 0.929 & $0 \cdot 636-1 \cdot 355$ & $0 \cdot 702$ & $0 \cdot 924$ & $0 \cdot 584-1 \cdot 462$ & $0 \cdot 736$ \\
\hline Nausea & $80(52 \cdot 3)$ & $540(51 \cdot 2)$ & $1 \cdot 045$ & $0 \cdot 744-1 \cdot 467$ & 0.799 & 0.783 & $0 \cdot 483-1 \cdot 269$ & $0 \cdot 321$ \\
\hline Vomiting & $51(33 \cdot 3)$ & $326(30 \cdot 9)$ & $1 \cdot 118$ & $0 \cdot 780-1 \cdot 604$ & $0 \cdot 544$ & $1 \cdot 339$ & $0 \cdot 797-2 \cdot 251$ & $0 \cdot 271$ \\
\hline Haematemesis & $3(2 \cdot 0)$ & $18(1 \cdot 7)$ & $1 \cdot 152$ & $0.335-3.959$ & $0 \cdot 822$ & $0 \cdot 819$ & $0 \cdot 178-3 \cdot 771$ & 0.797 \\
\hline Abdominal pain & $13(8 \cdot 5)$ & $136(12 \cdot 9)$ & 0.627 & $0 \cdot 346-1 \cdot 139$ & $0 \cdot 125$ & 0.537 & $0 \cdot 265-1 \cdot 089$ & $0 \cdot 085$ \\
\hline Diarrhoea & $43(28 \cdot 1)$ & $268(25 \cdot 4)$ & $1 \cdot 148$ & $0 \cdot 786-1 \cdot 677$ & $0 \cdot 475$ & $1 \cdot 207$ & $0 \cdot 785-1 \cdot 857$ & $0 \cdot 391$ \\
\hline
\end{tabular}

OR, Odds ratio; CI, confidence interval, s.D., standard deviation.

* Multivariate logistic regression analyses were performed.

\section{Study}

Jiao et al., $2011^{[3]}$

Zhang et al., 2011 $211^{[21]}$

Liu et al., 2012 ${ }^{[34]}$

Gong et al., $2013^{[42]}$

Wang et al., 2013

Cui et al., 2014

Liang et al., 2014

Li et al., $2014^{[36]}$

Sun et al., $2014^{[27]}$

Zhang et al., 2014 ${ }^{[23]}$

Ding et al., 2014

Hu et al., $2014^{[40]}$

Fixed effect model

Random effects model

Reterogeneity: squared $=97.5 \%$,
Events Total

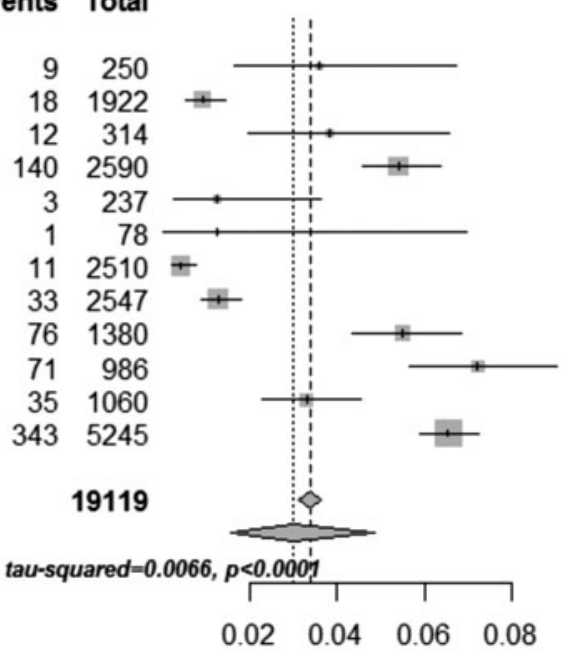

Proportion $\quad 95 \%-\mathrm{Cl}$ W(fixed) $\mathrm{W}$ (random)

$\begin{array}{rrrr}0.036[0.017 ; 0.067] & 1.3 \% & 7.8 \% \\ 0.009[0.006 ; 0.015] & 10.1 \% & 8.8 \% \\ 0.038[0.020 ; 0.066] & 1.6 \% & 8.0 \% \\ 0.054[0.046 ; 0.063] & 13.5 \% & 8.9 \% \\ 0.013[0.003 ; 0.037] & 1.2 \% & 7.8 \% \\ 0.013[0.000 ; 0.069] & 0.4 \% & 6.1 \% \\ 0.004[0.002 ; 0.008] & 13.1 \% & 8.9 \% \\ 0.013[0.009 ; 0.018] & 13.3 \% & 8.9 \% \\ 0.055[0.044 ; 0.068] & 7.2 \% & 8.7 \% \\ 0.072[0.057 ; 0.090] & 5.2 \% & 8.7 \% \\ 0.033[0.023 ; 0.046] & 5.5 \% & 8.7 \% \\ 0.065[0.059 ; 0.072] & 27.4 \% & 8.9 \% \\ & & & \\ \mathbf{0 . 0 3 4}[0.031 ; 0.037] & \mathbf{1 0 0} \% & - \\ \mathbf{0 . 0 3 0}[0.016 ; 0.049] & -- & \mathbf{1 0 0} \%\end{array}$

meta-analysis. The sample sizes ranged from 78 to 5245, yielding altogether 19119 study subjects (Fig. 3). This yielded an overall seroprevalence of $3 \cdot 0 \%$ (95\% CI 1.6-4.9) (Table 3). Respectively 3.3\% of female and $3 \cdot 3 \%$ of male subjects were detected as positive for SFTSV-specific IgG antibody, revealing no significant association between antibody response and gender. SFTSV seroprevalence appeared to be comparable in groups aged $<70$ years, but significantly increased in subjects aged $\geqslant 70$ years. Seroprevalence was slightly enhanced in high-risk areas $(3 \cdot 9 \%, 95 \%$ CI $2 \cdot 5-5 \cdot 5)$ than in low-risk areas $(2 \cdot 6 \%, 95 \%$ CI $0 \cdot 9-5 \cdot 1)$. However, this difference failed to reach significance. When the pooled data on seroprevalence was compared with case incidence and case fatality, a preference to develop clinical 
Table 3. The seropositive rates of SFTSV in healthy subjects and estimated SFTS case incidence and case-fatality rate

\begin{tabular}{|c|c|c|c|c|c|c|}
\hline Variable & $\begin{array}{l}\text { Seroprevalence* } \\
\text { mean }(95 \% \mathrm{CI})\end{array}$ & $\begin{array}{l}P \\
\text { value } \dagger\end{array}$ & $\begin{array}{l}\text { Case incidence } \\
\text { ratet }\left(\text { per } 10^{5}\right)\end{array}$ & $P$ value $\dagger$ & $\begin{array}{l}\text { Case-fatality } \\
\text { rate }(\%)\end{array}$ & $P$ value \\
\hline Age, years & & $<0 \cdot 001$ & & $<0 \cdot 001$ & & $<0 \cdot 001$ \\
\hline$<40$ & $3 \cdot 3(1 \cdot 1-6 \cdot 5)$ & & $0 \cdot 010$ & & $6 \cdot 8(4 / 59)$ & \\
\hline $40-50$ & $3 \cdot 4(1 \cdot 3-6 \cdot 5)$ & & $0 \cdot 121$ & & $4 \cdot 9(8 / 165)$ & \\
\hline $50-60$ & $2 \cdot 3(0 \cdot 3-4 \cdot 9)$ & & $0 \cdot 281$ & & $9 \cdot 2(24 / 261)$ & \\
\hline $60-70$ & $3 \cdot 7(1 \cdot 5-6 \cdot 9)$ & & 0.575 & & $15 \cdot 1(35 / 392)$ & \\
\hline$\geqslant 70$ & $7 \cdot 1(5 \cdot 8-8 \cdot 6)$ & & 0.592 & & $19 \cdot 5(55 / 282)$ & \\
\hline Sex & & 0.921 & & $0 \cdot 465$ & & $0 \cdot 261$ \\
\hline Male & $3 \cdot 3(1 \cdot 4-5 \cdot 9)$ & & $0 \cdot 136$ & & $14 \cdot 3(133 / 931)$ & \\
\hline Female & $3 \cdot 3(1 \cdot 2-6 \cdot 6)$ & & $0 \cdot 153$ & & $12 \cdot 5(124 / 989)$ & \\
\hline Area§ & & $<0 \cdot 001$ & & $<0 \cdot 001$ & & $0 \cdot 422$ \\
\hline High endemic & $3 \cdot 9(2 \cdot 5-5 \cdot 5)$ & & 0.567 & & $13 \cdot 9(161 / 1159)$ & \\
\hline Middle endemic & $2 \cdot 6(0 \cdot 9-5 \cdot 1)$ & & $0 \cdot 067$ & & $12 \cdot 6(96 / 761)$ & \\
\hline Recruitment year & & $<0 \cdot 001$ & & $<0.001$ & & 0.030 \\
\hline 2010 & $2 \cdot 2(0 \cdot 7-4 \cdot 5)$ & & $0 \cdot 004$ & & $25 \cdot 3(61 / 241)$ & \\
\hline 2011 & $3 \cdot 1(0 \cdot 8-6 \cdot 7)$ & & 0.034 & & $13.9(71 / 510)$ & \\
\hline 2012 & $3 \cdot 0(0 \cdot 3-8 \cdot 2)$ & & $0 \cdot 046$ & & $10 \cdot 4(59 / 569)$ & \\
\hline 2013 & $6 \cdot 5(5 \cdot 6-7 \cdot 4)$ & & 0.052 & & $8.4(37 / 439)$ & \\
\hline Total & $3 \cdot 0(1 \cdot 6-4 \cdot 9)$ & & $0 \cdot 144$ & & $12 \cdot 2(257 / 1920)$ & \\
\hline
\end{tabular}

*SFTSV-specific IgG antibody-positive rate by meta-analysis.

$\dagger$ Using $\chi^{2}$ test

$\$$ The incidence of SFTSV infections per 100000 of the total population.

$\S$ High endemic region: the province which reported SFTS case incidence of $\geqslant 0 \cdot 5 / 10^{5}, n=2$; medium-risk region: incidence rate $<0 \cdot 5 / 10^{5}, n=5$.

disease was found in female and elderly patients, while a higher frequency of fatal outcome was found in male and elderly patients, on the premise of similar subclinical levels. The forest plots are summarized in Supplementary Figure S6.

\section{DISCUSSION}

In the current study, by applying descriptive analysis on an integrated dataset of a large case series, we have presented a reliable clinical picture and laboratory abnormalities of SFTSV infection that can be used to facilitate the clinical diagnosis. A verified CFR of $12 \cdot 2 \%$ was estimated, significantly higher than that obtained from national reporting data $[2$, 10]. Based on verified case mortality, older age and longer delay from disease onset to hospitalization were found to be associated with fatal outcome. Meta-analysis on the published seroprevalence data revealed a pooled seroprevalence of $3 \%$ from apparently healthy subjects, suggesting the existence of an iceberg phenomenon in SFTSV infection. Although males and females were equally exposed to the virus, females had a higher tendency to develop symptomatic disease, while males more easily progressed to fatal outcome, therefore gender-related heterogeneity in determining disease outcome was proposed.

Until recently, the clinical indicators that could be utilized for the diagnosis of SFTS were restricted to fever, thrombocytopenia and leukocytopenia [16]. Here, we found additional syndromes, including anorexia, myalgia, chill and lymphadenopathy, which when presenting with high frequencies at early infection can be applied for SFTS recognization. Lymphadenopathy is especially useful for the differential diagnosis from common viral infection, and other tick-borne infectious disease, such as human anaplasmosis and rickettsiosis. In addition to WBC and PLT, commonly seen laboratory indicators, including LDH, AST, ALT and CK were identified as abnormal in a high proportion at acute infections. Elevated LDH and AST levels were overrepresented, even more so than thrombocytopenia and leukocytopenia. This finding is consistently identified throughout all the studied years and most of the endemic regions, therefore we recommend the utility of these indicators as supportive evidence when making the clinical 
diagnosis, before the molecular detection of SFTSV can be made in clinical practice.

Since the discovery of SFTS, there have been numerous reports on its ability to cause death, which is considered to be crucial for both evaluation of the health toll on the public and exploration of the disease pathogenesis mechanism. However, the data reported so far varies marginally across clinical studies. The estimation from national data of 2010-2012 and 2010 2013 calendar year was around $8 \%[2,15]$. The current meta-analysis, based on the integrated dataset, revealed a verified estimation $(12 \cdot 2 \%)$ that was significantly higher than the nationally reported data. Inter-province comparison revealed the nationally reported CFR has been marginally underestimated in Henan province, where over one third of the total cases were reported [2]. Further investigation revealed that this low rate is not a feature of the infection itself in local areas, rather, it is likely to result from a failure to account for the patients who were misreported as being alive by sentinel hospitals. The cultural custom in local areas is that individuals choose to die in their own home instead of in a hospital, therefore those patients with adverse disease tended to stop therapy against medical advice and were hence misreported as alive to the CISDCP system. Taking these unreported deaths into account, the widely cited CFR of around $8 \%$ is a substantial underestimate of the real situation. Since this custom also exists in other rural area of China, we postulate the actual case fatality might be even higher than the $12 \cdot 2 \%$, which, however, cannot be inferred from the current research. On the other hand, the significant interprovince difference of CFR that was calculated from nationally reportable data is not real [2], instead, the inter-province CFR was of a comparable level although the seroprevalence and case incidence varied marginally across provinces.

The iceberg phenomenon of SFTS has been indicated by seroprevalence in apparently healthy populations. Here by meta-analysis on available data, we have acquired a combined seroprevalence of $3 \%$ in a population residing in an endemic region, which shows inter-province difference. Significant positive correlation between seroprevalence rate and case incidence rate were observed when considered geographically, indicating the usefulness of the seroprevalence data at the population level as a reliable indicator for future infection occurrence. An increasing seropositive rate has been observed since 2010 [21, 33], indicating an accumulating exposure to the virus. The accumulation of subclinical infection, on the other hand, led to no marginal decrease of the reportable case incidence. One explanation is that the subclinical infection offers no persistent effective protection from the clinical disease. The other possibility is that the diagnostic capacity of the infection has been increased, thus resulting in a wider recognition and reporting of the disease.

With the premise of similar exposure to SFTSV between gender groups, a preference for developing clinical disease was found in females, while a tendency of developing fatal outcome was observed in male patients. Although not reaching significance, this difference has been consistently observed from each of the five SFTS high-endemic provinces. We therefore propose a gender-specific biological difference which presumably plays a role in determining the disease outcome, instead of the previously mentioned hypothesis that women are more often involved in fieldwork, thereby having a high probability of exposure to the potential vectors that carry SFTSV [2, 15]. Previous studies have found that in comparison to males, females have a higher tendency to develop severe disease after viral infection, from which the differentially expressed steroid levels or gender-specific host immunity response might play roles [45]. Whether the same situation holds for SFTS infection deserves further investigation. Except for the elderly ( $\geqslant 70$ years), age seems have no influence on subclinical infection level. With the premise of similar exposure to SFTSV, an age-specific increase of case incidence and case fatality is therefore proposed. Consistent with this finding, older age was also demonstrated to be associated with higher risk of death based on individual data.

Substantial interests have developed in identifying risk factors for fatal outcome, yielding a long yet controversial list of the clinical manifestations and laboratory indicators, which, however, failed to be replicated in the current meta-analysis. Older age and longer delay to hospital admission were shown to be the only factors that were associated with death based on large sample size and adequate statistical analysis. Thrombocytopenia was merely found to be increased in patient mortality by univariate analysis; however, this association disappeared after multivariate adjustment. A similar association between low PLT counts has been obtained from our study as well as previous studies [10, 11, 13, 43]. According to the current findings, we postulate this to be a false association that was likely to be derived from the association 
between long delay before hospitalization and lower PLT counts.

In summary, this study represents a clinical characterization on the largest group of SFTS patients up to now. A higher than expected CFR was obtained. A wider spectrum of clinical index was suggested to be used to identify SFTSV infection, while the useful predictor for fatal outcome was found to be restricted. These findings might help improve the current knowledge on clinical diagnosis and recognizing patients with adverse outcome. Information on the age- and gender-specific risks of becoming infected and of developing fatal outcome might be used to guide mitigation efforts, such as vaccination and effective treatment in case of further development.

\section{SUPPLEMENTARY MATERIAL}

For supplementary material accompanying this paper visit http://dx.doi.org/10.1017/S0950268815002678.

\section{ACKNOWLEDGEMENTS}

This work was supported by the Natural Science Foundation of China (81222037, 81290344, 81473023), China Mega-Project for Infectious Diseases grant (2013ZX10004-202) and the Special Fund for Quarantine-Scientific Research in the Public Interest (201310076).

\section{DECLARATION OF INTEREST}

None.

\section{REFERENCES}

1. Yu XJ, et al. Fever with thrombocytopenia associated with a novel bunyavirus in China. New England Journal of Medicine 2011; 364: 1523-1532.

2. Liu K, et al. A national assessment of the epidemiology of severe fever with thrombocytopenia syndrome, China. Scientific Reports 2015; 5: 9679.

3. Liu Q, et al. Severe fever with thrombocytopenia syndrome, an emerging tick-borne zoonosis. Lancet Infectious Diseases 2014; 14: 763-772.

4. Kim KH, et al. Severe fever with thrombocytopenia syndrome, South Korea, 2012. Emerging Infectious Diseases 2013; 19: 1892-1894.

5. Takahashi T, et al. The first identification and retrospective study of severe fever with thrombocytopenia syndrome in Japan. Journal of Infectious Diseases 2014; 209: 816-827.

6. Bao CJ, et al. A family cluster of infections by a newly recognized bunyavirus in eastern China, 2007: further evidence of person-to-person transmission. Clinical Infectious Diseases 2011; 53: 1208-1214.

7. Chen H, et al. A cluster of cases of human-to-human transmission caused by severe fever with thrombocytopenia syndrome bunyavirus. International Journal of Infectious Diseases 2013; 17: e206-208.

8. Tang $\mathbf{X}$, et al. Human-to-human transmission of severe fever with thrombocytopenia syndrome bunyavirus through contact with infectious blood. Journal of Infectious Diseases 2013; 207: 736-739.

9. Wang Y, et al. Person-to-person asymptomatic infection of severe fever with thrombocytopenia syndrome virus through blood contact. Internal Medicine (Tokyo, Japan) 2014; 53: 903-906.

10. Deng B, et al. Clinical features and factors associated with severity and fatality among patients with severe fever with thrombocytopenia syndrome Bunyavirus infection in Northeast China. PLOS ONE 2013; 8: e80802.

11. Gai ZT, et al. Clinical progress and risk factors for death in severe fever with thrombocytopenia syndrome patients. Journal of Infectious Diseases 2012; 206: 1095-1102.

12. Zhang YZ, et al. Hemorrhagic fever caused by a novel Bunyavirus in China: pathogenesis and correlates of fatal outcome. Clinical Infectious Diseases 2012; 54: 527-533.

13. Liu W, et al. Case-fatality ratio and effectiveness of ribavirin therapy among hospitalized patients in china who had severe fever with thrombocytopenia syndrome. Clinical Infectious Diseases 2013; 57: 1292-1299.

14. Liu K, et al. Epidemiologic features and environmental risk factors of severe fever with thrombocytopenia syndrome, Xinyang, China. PLoS Neglected Tropical Diseases 2014; 8: e2820.

15. Ding F, et al. Epidemiologic features of severe fever with thrombocytopenia syndrome in China, 2011-2012. Clinical Infectious Diseases 2013; 56: 1682-1683.

16. Ministry of Health, China. Guideline for prevention and treatment of severe fever with thrombocytopenia syndrome (2010 version). Chinese Journal of Clinical Infectious Diseases 2011; 4: 193-194.

17. Zhang YZ, et al. Hemorrhagic fever caused by a novel tick-borne Bunyavirus in Huaiyangshan, China. Chinese Journal of Epidemiology 2011; 32: 209-220.

18. Zhang YY, et al. Clinical features of fever with thrombocytopenia syndrome: report of 16 cases. Chinese Journal of Clinical Infectious Diseases 2012; 5: 89-90.

19. Zhang YG, et al. Clinical characteristics and epidemiology of novel bunyavirus human infection cases in Dandong city, 2010-2012. Chinese Journal of Public Health 2013; 29: 201-204.

20. Zhang XL, et al. Nursing care of 15 patients infected by novel Bunyavirus. Chinese Nursing 2012; 47: 123-124.

21. Zhang WS, et al. Seroepidemiology of severe with thrombocytopenia syndrome bunyavirus in Jiangsu province. Disease Surveillance 2011; 26: 676-678.

22. Zhang SP, et al. Clinical analysis of 12 cases of SFTSV infected by novel bunyavirus. Jiangsu Medical Journal 2012; 38: 583-584. 
23. Zhang L, et al. Antibodies against severe fever with thrombocytopenia syndrome virus in healthy persons, China, 2013. Emerging Infectious Diseases 2014; 20: 1355-1357.

24. Yu B, et al. Epidemiological study on data involving 61 hospitalized cases with Huaiyangshan hemorrhagic fever in Wuhan. Chinese Journal of Epidemiology 2012; 33: 124-125.

25. Xia YJ, et al. Clinical analysis of 12 cases associated hemophagocytic comprehensive with novel bunyavirus syndrome infected. Journal of Clinical Internal Medicine 2013; 30: 487-488.

26. Wang L, et al. Surveillance and analysis of severe fever with thrombocytopenia syndrome in Zibo City. Modern Preventive Medicine 2013; 40: 3471-3474.

27. Sun JM, et al. Seroprevalence of severe fever with thrombocytopenia syndrome virus in southeastern China and analysis of risk factors. Epidemiology and Infection 2015; 143: 851-856.

28. Sun J, et al. Epidemiological characteristics of severe fever with thrombocytopenia syndrome in Zhejiang Province, China. International Journal of Infectious Diseases 2014; 25: 180-185.

29. Song LH, et al. Epidemiological characteristics and control strategy of patients infected by SFTSV in Dalian, China. Chinese Journal of Pest Control 2012; 28: 1325-1327.

30. Shen LM, et al. 13 cases of the new virus infection nursing experience in acute stage. Zhejiang Medical Journal 2013; 35: 2222-2224.

31. Qiu YQ. Nursing care of patients infected by novel bunyavirus. Chinese Journal of Practical Nursing 2012; 28: 78-79.

32. Long ZG, et al. Clinical analysis of 11 cases with a new variety of bunyavirus infection. Journal of Internal Intensive Medicine 2011; 17: 340-342.

33. Liu Y, et al. Person-to-person transmission of severe fever with thrombocytopenia syndrome virus. Vector Borne and Zoonotic Diseases (Larchmont, NY) 2012; 12: $156-160$.

34. Liu L, et al. Epidemiologic analysis on severe fever with thrombocytopenia syndrome in Hubei province, 2010. Chinese Journal of Epidemiology 2012; 33: 168-172.
35. Liang S, et al. Seroprevalence and risk factors for severe fever with thrombocytopenia syndrome virus infection in Jiangsu Province, China, 2011. American Journal of Tropical Medicine and Hygiene 2014; 90: 256-259.

36. Li Z, et al. Seroprevalence of antibodies against SFTS virus infection in farmers and animals, Jiangsu, China. Journal of Clinical Virology 2014; 60: 185-189.

37. Jiao Y, et al. Preparation and evaluation of recombinant severe fever with thrombocytopenia syndrome virus nucleocapsid protein for detection of total antibodies in human and animal sera by double-antigen sandwich enzyme-linked immunosorbent assay. Journal of Clinical Microbiology 2012; 50: 372-377.

38. Huang X, et al. Epidemiological and etiological characteristics of fever, thrombocytopenia and leukopenia syndrome in Henan Province, China, 2011-2012. PLoS ONE 2014; 9: e91166.

39. Hu JL, et al. Clinical and epidemiological characteristics on 20 cases of SFTSV infection. Chinese Journal of Zoonoses 2012; 28: 302-305.

40. Hu C, et al. The severe fever with thrombocytopenia syndrome bunyavirus (SFTSV) antibody in a highly endemic region from 2011 to 2013: a comparative serological study. American Journal of Tropical Medicine and Hygiene 2015; 92: 479-481.

41. Han MF, et al. Clinical features and nursing care of patients infected by SFTSV in Zhoushan area. Journal of Modern Nursing 2013; 19: 1901-1903.

42. Gong LF, et al. Prevalence and homology analysis on human and animals severe fever with thrombocytopenia syndrome virus infection in Yantai of Shandong province. Chinese Journal of Epidemiology 2014; 35: 524-527.

43. Ding YP, et al. Prognostic value of clinical and immunological markers in acute phase of SFTS virus infection. Clinical Microbiology and Infection 2014; 20: 870-878.

44. Cui F, et al. Clinical and epidemiological study on severe fever with thrombocytopenia syndrome in Yiyuan County, Shandong Province, China. American Journal of Tropical Medicine and Hygiene 2013; 88: 510-512.

45. Fish EN. The X-files in immunity: sex-based differences predispose immune responses. Nature Reviews Immunology 2008; 8: 737-744. 